\title{
Reflets
}

Revue d'intervention sociale et communautaire

\section{Recentrer la pratique communautaire sur les droits humains : un début de réflexion}

\section{Vincent Greason}

Volume 21, numéro 2, automne 2015

URI : https://id.erudit.org/iderudit/1035441ar

DOI : https://doi.org/10.7202/1035441ar

Aller au sommaire du numéro

Éditeur(s)

Reflets, Revue d'intervention sociale et communautaire

ISSN

1203-4576 (imprimé)

1712-8498 (numérique)

Découvrir la revue

Citer cet article

Greason, V. (2015). Recentrer la pratique communautaire sur les droits humains : un début de réflexion. Reflets, 21(2), 229-240.

https://doi.org/10.7202/1035441ar d'utilisation que vous pouvez consulter en ligne. 


\section{Recentrer la pratique communautaire sur les droits humains : un début de réflexion}

\section{Vincent Greason}

Table ronde des organismes volontaires d'éducation populaire de l'Outaouais (TROVEPO)

"Considérés dans leur ensemble, les droits humains imposent des exigences dans l'organisation de la société : ils sont porteurs d'un projet de société fondé sur l'égalité, la liberté et la solidarité. Ils définissent également ce qu'un individu est en droit de réclamer avant tout de l'État, mais aussi des autres composantes de la société. " Ligue des droits et libertés, 2010

Cela m'a frappé comme une tonne de briques. Collé sur les portes du tout nouveau pavillon des Sciences sociales de l'Université d'Ottawa, un autocollant indiquant " Heures d'affaires : 6 h $30-$ $23 \mathrm{~h}$ ». Existe-t-il un symbole plus décourageant de la marchandisation de l'éducation que ce petit autocollant vantant les " heures d'affaires " du pavillon même où l'université est en train de former une prochaine génération de travailleuses et de travailleurs sociaux?

Les organisations communautaires ne sont pas imperméables à la tendance néolibérale de tout marchandiser, bien au contraire. Issus du mouvement de transformation sociale des années 70, les premiers groupes populaires furent fréquentés par des membres (des citoyennes et des citoyens engagés) qui se sont donné des véhicules collectifs de luttes. Depuis les vingt dernières années, les membres ont cédé leur place aux usagers et usagères de services 
qui sont tranquillement devenus des clientes ou clients ou des consommatrices ou consommateurs de services (St-Amand, 2000).

Suivant la trajectoire imposée par le néolibéralisme, de plus en plus d'organismes communautaires adoptent des pratiques de clientélisme, axées sur la fourniture de services à des consommatrices ou consommateurs (Greason, 2010). Remettant en question cette trajectoire, le présent article propose de revenir à une approche mettant de l'avant une préoccupation des droits humains. La citoyenne et le citoyen sont au centre de cette approche. Une précision s'impose toutefois. Il existe au Québec un type d'intervention communautaire, dit " de la défense collective des droits» et reconnu par le gouvernement. Parmi les groupes ainsi reconnus se trouvent ceux qui interviennent pour défendre des droits spécifiques, entre autres, ceux des locataires, des personnes en chômage, des personnes assistées sociales ou des consommatrices et consommateurs. Cet article n'inclut pas de tels groupes. Il explore plutôt comment le milieu communautaire dans son ensemble peut se doter des pratiques qui remettent en question la tendance lourde du clientélisme et de la marchandisation en instaurant celles axées sur la liberté, l'égalité et la solidarité, soit les trois valeurs nécessaires au respect des droits humains.

\section{Un bref retour historique - L'État et les protections sociales}

Adoptée par les Nations Unies en 1948, la Déclaration universelle des droits de l'homme confirme que les droits humains doivent être au cœur de l'action gouvernementale. La Déclaration est complétée par l'adoption de deux pactes des Nations Unies : le Pacte international relatif aux droits économiques, sociaux et culturels (PIDESC,1966) et le Pacte international relatif aux droits civils et politiques (PIDCP, 1966). Tous les grands instruments de promotion des droits humains ont ceci en commun : ils émergent d'un environnement politique dominé par le keynésianisme. Ainsi comprises, les mesures de protection sociale, telles que les régimes d'aide sociale, de santé et 
d'éducation publiques, d'aide juridique, de normes de travail, de logement social ou de protection d'accidentés de travail, répondent aux obligations de l'État en matière des droits humains.

Bien sûr, cette réponse étatique n'est pas tombée du ciel. Au Québec et au Canada, ce sont les mouvements sociaux, dont le communautaire, qui ont forcé l'État à reconnaître ses obligations dans le domaine du social. Ce sont eux qui arrachent morceau par morceau les éléments qui forment la "sécurité sociale " (services publics et programmes sociaux). L'universalité, la gratuité et le caractère public des services en éducation et en santé découlent des revendications provenant d'un mouvement social militant et mobilisé, et particulièrement de sa composante syndicale. Grâce aux années de pression et de mobilisation, qui ont débuté dans les années 1950, des gains tangibles en matière de l'avancement des droits économiques et sociaux sont enregistrés pendant les années 1960 et 1970.

\section{Des pas en arrière}

Dans les décennies 1970-1980, une brèche se produit dans le consensus social concernant le rôle de l'État. L'État keynésien, dit " dispensateur de grands programmes sociaux ", s'estompe au profit d'un nouvel "État accompagnateur ». Le néolibéralisme s'installe avec pour conséquence que la place accordée aux droits régresse, tant au niveau provincial que fédéral, et indépendamment du parti politique au pouvoir.

Suivant le chemin emprunté par les néolibéraux d'ailleurs (É-U, Angleterre, Australie), notre État accompagnateur restructure la manière de concevoir et d'offrir des services et des programmes. Pour reprendre une image de Madame JérômeForget, l'État doit "piloter plutôt que ramer ». ${ }^{1}$ Le nouvel État offre moins de services directs à la population, et les services publics restants sont moins accessibles et offerts moins largement. Adieu au principe de l'universalité; bonjour au clientélisme, au ciblage, aux "services de base ", à la privatisation, à la philanthropie... Bienvenue au communautaire! 


\section{Le communautaire face au néolibéralisme}

Né dans la contestation des années 70, le déploiement du communautaire québécois suit la transformation de la société dans laquelle il évolue. D'abord un mouvement démocratique, contrôlé par les membres, le milieu communautaire a été contestataire envers le capital (TROVEPO, 2014). En effet, les premiers groupes de citoyens naissent en résistance aux projets d'urbanisation de grands centres-ville (Montréal, Québec, Trois-Rivières, Hull [aujourd'hui Gatineau]). S'inspirant des pratiques d'éducation populaire et étant relativement politisés, les premiers groupes, composés de citoyennes et de citoyens directement touchés par les enjeux, s'opposent à la construction d'autoroutes dans les quartiers populaires et à l'expropriation des logements pour la construction des tours de bureaux. Ils comprennent que leurs droits sont brimés afin de privilégier les besoins des spéculateurs. L'ennemi, c'est le capital.

Mais rapidement, le communautaire modifie la cible de sa mobilisation. La nouvelle cible, c'est l'État. Pour le communautaire, le pouvoir public devient progressivement un bailleur de fonds, une source de financement des organismes qui sont en pleine expansion : d'abord pour répondre aux "besoins " toujours croissants en provenance de "la communauté ", ensuite pour répondre aux mandats accrus refilés de l'État et enfin pour payer le nombre grandissant de personnes salariées nécessaires pour répondre aux nouveaux besoins et mandats. Entre temps, la base militante des premiers groupes se transforme. Les membres deviennent les usagères ou usagers qui deviennent les clientes ou clients. Les groupes, devenus organismes, puis entreprises, sont tous débordés et, pour bien accomplir leur travail, demandent la reconnaissance et le financement compensatoire.

Pendant ce temps, lui-même en pleine transformation, l'État cherche à se dégager de ses responsabilités, entre autres et de façon particulière, dans le domaine du social. Il est heureux de confier ses responsabilités aux "partenaires " qui ont une certaine expertise, et surtout qui coûtent moins chers. Puisque les groupes 
communautaires se conforment de plus en plus aux priorités de l'État catalyseur, celui-ci se montre progressivement ouvert à les reconnaitre, d'abord par le financement et ensuite par l'octroi de mandats.

De même, ces nouvelles orientations redéfinissent les rapports entre organismes communautaires et État, mais aussi entre citoyennes ou citoyens et État ainsi qu'entre citoyennes ou citoyens et organismes communautaires. Ceux-ci, lorsqu'ils conviennent de rendre les services qui relèvent de la responsabilité gouvernementale, deviennent en quelque sorte l'interface entre l'État et les citoyennes ou citoyens.

\section{Impact sur les droits}

La transition vers le néolibéralisme a un effet important sur la réalisation des droits humains, puisque l'approche clientéliste ou par cibles convient particulièrement mal à celle-ci. S'il va de soi que les droits s'appliquent universellement - j'ai des droits parce que je suis humain; mes droits sont identiques aux vôtres parce que nous sommes tous deux humains - il n'est pas étonnant que le démantèlement néolibéral de l'universalité des programmes et services publics ait un impact sur les droits. Et de fait, le discours néolibéral trahit ces derniers, particulièrement celui des droits économiques et sociaux. Repris par le néolibéralisme, le discours sur les droits s'accompagne d'un autre discours de plus en plus insistant sur les responsabilités. Un détenteur de droits a dorénavant une obligation parallèle d'être responsable.

Autant le néolibéralisme influence les politiques publiques, autant il influence les pratiques des groupes communautaires. Le droit à un niveau de vie suffisant devient donc conditionnel au fait que les citoyennes ou citoyens soient responsables et qu'ils travaillent; c'est la prémisse même des stratégies gouvernementales qui donnent naissance à des groupes communautaires en employabilité, où on apprend comment se laver, se lever de bonne heure ou s'habiller. Dans le domaine du logement social, le droit au 
logement cède tranquillement le pas à la gestion sociale : de plus en plus, l'accès à certains logements sociaux devient conditionnel à l'engagement de la personne de se reprendre en main par la signature d'un code de vie, alors qu'elle cherche simplement à améliorer ses conditions de vie. Si la personne respecte les engagements pris avec l'organisme, elle peut demeurer dans un logement avec soutien communautaire. Si elle ne se responsabilise pas, une rupture de la relation qui lie ces individus à leur communauté s'établit et elle risque alors de perdre son logement. Pour encadrer cela, un nouveau contrat de location apparait : le «bail thérapeutique »(Ligue des droits et libertés, 2010, p. 49)!

\section{Remettre le citoyen et citoyenne au cœur des pratiques communautaires}

Pour contrer l'approche clientéliste, le défi consiste à retrouver, collectivement, une façon de faire (une pratique) qui fait avancer l'égalité, la liberté et la solidarité au sein des organismes communautaires. Autrement dit, on doit appliquer à l'intervention communautaire les propos émis au début du présent article. Si les droits humains imposent des exigences dans l'organisation de la société, vouloir s'y prêter en exige autant dans l'organisation du groupe communautaire.

Voici quelques pistes pour aider un organisme communautaire qui voudrait recentrer ses pratiques sur la citoyenne ou le citoyen. Ce faisant, le groupe deviendra un lieu où la citoyenne ou le citoyen puisse vivre une expérience de liberté, d'égalité et de solidarité, les trois valeurs qui sous-tendent une société respectueuse des droits humains. Nous reconnaissons l'apport d'un projet de recherche-action de la Ligue des droits et libertés auquel nous étions grandement impliqués et dont le Guide est le fruit (Ligue des droits et libertés, 2010). Le projet et la formation qui l'accompagnait ont permis de constater à quel point considérer les droits humains et agir en conséquence peut colorer les pratiques de n'importe quel organisme communautaire, et ce, 
sinon positivement (dans le sens de les faire avancer), au moins dans le sens de ne pas les brimer ou de les faire reculer davantage!

\section{Quelques points de repère}

Tout groupe communautaire existe pour une raison précise, celle de réaliser sa mission. Celle-ci se trouve souvent dans un énoncé intégré au règlement de régie interne. Un premier lieu pour voir comment un groupe peut adopter une approche de droits humains est dans un examen de sa mission. La citoyenne ou le citoyen y est-il au cœur? En marge? Absente ou absent? La mission est-elle rédigée de manière à refléter nommément la préoccupation d'une prise en charge citoyenne. Cette préoccupation est-elle reflétée dans le choix de langage (" clientèle ») et de verbes, actifs ou passifs?

Le premier contact entre une personne et le groupe influence souvent la nature de la relation qui va suivre. Peu importe le type de service offert, la perspective citoyenne peut être intégrée aux pratiques d'accueil de tout groupe communautaire. Certaines approches peuvent s'apparenter davantage au clientélisme, d'autres moins. Considérons deux exemples concrets du clientélisme extraits du Guide :

- "Une personne "en crise" arrive dans mon organisme. Chez nous, on refuse de travailler en situation d'agressivité, alors on lui indique une autre ressource, mieux outillée que nous, pour répondre à sa crise et on lui demande de revenir nous voir dans un couple de jours. Puis, discrètement, on appelle la police, au cas où... ";

- «Chez nous, la procédure est claire. La personne arrive. Pour être certain qu'elle est à la bonne place - on ne veut pas qu'elle perde son temps - , on lui présente la mission, le fonctionnement et les règles de l'organisme. Sur cette base, on lui demande ensuite comment notre organisme peut lui être utile." 
De tels exemples permettent de réfléchir sur des enjeux fondamentaux en matière des droits humains. Accueille-t-on la personne qui arrive dans le groupe comme citoyenne ou citoyen ayant des besoins multiples? La personne répond-elle aux besoins du groupe ou le groupe aux besoins humains? La personne a-telle l'impression d'être importante ou dérangeante? Y a-t-il une meilleure façon (du point de vue des droits humains) d'organiser l'accueil?Y a-t-il des pratiques à éviter ou même à bannir?

D'autres enjeux se présentent quant aux pratiques relatives à l'accès aux services. Alors que la vaste majorité des personnes se présente dans un organisme communautaire pour obtenir un service, la question se pose : l'organisme peut-il tout faire? S'il ne peut répondre à toutes les demandes, doit-il établir des limites? Si oui, selon quels critères?

Répondre à de telles questions soulève la question de l'offre de services et celle d'enjeux qui peuvent brimer ou bien respecter les droits des personnes qui se présentent dans un groupe. Considérons deux exemples réels, identifiés dans l'enquête de la Ligue :

- "Chez nous, on prend le temps pour bien connaitre les personnes et pour s'assurer que notre groupe répond à leurs attentes. On s'assoit régulièrement avec elles, autant dans les moments formels qu'informels. Juste pour jaser et pour faire un suivi. Ce n'est pas toujours facile, mais c'est comme ça qu'on fonctionne. On essaie de respecter la personne. ";

- «Nous avons une rencontre d'équipe toutes les semaines. Lors de cette rencontre, les travailleuses passent en revue chacun de nos clients. Vous n'avez aucune idée de l'importance de ces rencontres. On y découvre des choses qu'on n'aurait jamais crues sur des personnes. L'idée ce n'est pas de faire du commérage, c'est d'améliorer nos services.»

L'enjeu fondamental des deux exemples touche le respect des droits humains versus le contrôle social. Dépendant des pratiques mises en place, un organisme peut respecter le droit à la confidentialité et à la vie privée, le droit à l'information, le droit à des services de qualité, le droit à l'accompagnement, le droit à un consentement libre et éclairé. Dans la mesure où telle est la 
voie choisie, l'organisme travaille dans une optique qui promeut en son sein les valeurs d'égalité, de liberté et de solidarité.

Par ailleurs, d'autres façons de faire instaurent des pratiques de contrôle. Celles-ci mettent les personnes dans une situation de non-respect de leur dignité ou de leurs droits. Le contrôle social place l'organisme communautaire dans une situation de pouvoir vis-à-vis des personnes qui le fréquentent, et ce, par rapport à ce qu'elles peuvent ou ne peuvent pas faire ou par rapport à la façon dont elles doivent se comporter.

D'un tout autre registre, le milieu communautaire n'est rien s'il n'est pas un lieu d'implication citoyenne. Or, dans une perspective de droits humains, la question se pose : Comment canaliser cette participation pour aboutir dans des actions concrètes facilitant la mise en œuvre de la liberté, de l'égalité et de la solidarité?

Certains organismes se tournent encore vers les citoyens pour déterminer leur programmation, soit entièrement ou partiellement. Planifier les activités du groupe en fonction des besoins exprimés par les participantes et participants demeure sans doute la meilleure manière de mettre la citoyenne ou le citoyen au cœur du groupe. Cependant, la pratique d'autodétermination citoyenne du communautaire est de plus en plus remise en question, notamment par le mode de financement qu'est l'entente de service. Celui-ci implique que le bailleur de fonds impose ses exigences sur le groupe quant aux services rendus et aux résultats à obtenir. Cette façon de faire mine les pratiques citoyennes et détourne souvent la mission éducative du communautaire.

L'intrusion des bailleurs de fonds dans la vie interne du groupe communautaire dénature les pratiques démocratiques de l'intervention communautaire. Elle introduit une certaine professionnalisation et priorise l'efficience et l'efficacité de l'intervention au détriment d'autres aspects de l'intervention communautaire. De plus, cette intrusion a tendance à porter un resserrement de l'action du groupe, parce qu'il est financé pour faire ceci et non pas cela. Par conséquent, la conception du groupe comme un espace de liberté, d'égalité et de solidarité peut être remise en question face aux choix déchirants comme ceux-ci : 
- Un organisme à mission déterminée donnera-t-il du service à une personne qui vit une double problématique? Une femme, ayant des problèmes de santé mentale, fera-t-elle fuir d'autres femmes susceptibles de venir dans le groupe?

- Un organisme refusera-t-il quelqu'un parce qu'il n'a pas les moyens de fournir l'accommodement nécessaire (interprètes, lieux accessibles, etc.) à la participation de la personne?

- Que faire avec la personne qui a proféré des menaces ou qui est "dérangeante »? Avec celle qui a porté plainte contre l'organisme ou contre un travailleur ou travailleuse de l'organisme?

Poser la question des droits humains et de leur place au sein des pratiques communautaires, c'est être plongé au cœur de la vraie vie. Parce que dans la vraie vie, les organismes communautaires sont souvent appelés à intervenir auprès des personnes qui n'ont pas eu la vie facile, mais qui ont quand même des droits. Aborder les pratiques communautaires sous l'angle des droits des personnes nous plonge dans le contexte quotidien des organismes communautaires, où les relations interpersonnelles sont parfois compliquées, sinon carrément conflictuelles, où les besoins des personnes sont souvent immédiats, où la survie d'une personne est l'enjeu et où les intervenantes ou intervenants sont trop souvent débordés.

Poser la question des droits humains par rapport aux pratiques communautaires exige un recentrage de celles-ci sur les besoins des citoyens et des citoyennes. En même temps, c'est un rappel à l'ordre. Au nom de la citoyenne et du citoyen, l'intervention communautaire doit répondre aux exigences autres que la performance et l'efficience. 


\section{Pour conclure - Retrouver les pratiques de transformation sociale}

Le mouvement communautaire a été une force collective de transformation sociale au Québec. C'est par la mobilisation collective qu'il a fait avancer les droits humains. Pour bâtir une société plus juste et plus égalitaire, de l'action collective est requise. Une pratique de groupe qui se limite à l'offre d'un service individuel ne s'inscrit pas dans une perspective de changement social.

Adopter une perspective d'intervention axée sur l'avancement des droits humains repose sur une conception d'action communautaire qui le définit comme un acteur politique. S'inscrivant en faux par rapport à la conception néolibérale dominante, des pratiques de droits humains remettront l'action collective au cœur de l'intervention communautaire. Retrouver des pratiques de droits humains, axées sur une conception du communautaire comme un espace de liberté, d'égalité et de solidarité, n'est-il pas un moyen pour renverser la vapeur d'un clientélisme qui amène ailleurs?

\section{Note}

Propos inspirés d'Osbourne et Gabler (1996), Steering Not Rowing.

\section{Bibliographie}

GREASON,Vincent (2010). Le communautaire québécois : Où sont les membres?, réf. du 22 septembre 2015, http://trovepo.org/documents/O\%C3\%B9sontlesmembres.pdf

LAMARCHE, Lucie (1996). «L'État désétatisé et ses fonctions sociales », dans Sylvie Paquerot (dire.),

L'État aux orties? Mondialisation de l'économie et rôle de l'État, Montréal, Les éditions Écosociété, p. 124-133.

LEBEL, Georges A. (2010). «La gestion du “communautaire” et la gouvernance néolibérale », Nouveaux cahiers du socialisme, $\mathrm{N}^{\circ} 3$, Hiver 2010, p. 118-131. 
LIGUE DES DROITS ET LIBERTÉS (2010). Pour tous les groupes communautaires : L'exercice des droits, un projet de société? - Un guide/questionnaire portant sur les droits humains et les pratiques du milieu communautaire, réf. du 22 septembre 2015, http://liguedesdroits.ca/wp-content/fichiers/ guide-final.pdf

LIGUE DES DROITS ET LIBERTÉS (2013). Tu ne vas pas m'appauvrir pour t'enrichir - Rapport sur les droits de la personne au Québec et au Canada, réf. du 22 septembre 2015, http://liguedesdroits. $\mathrm{ca} /$ wp-content/fichiers/rappot-droits-humains-web.pdf

ST-AMAND, Nérée (2000). "Des noms qui en disent long... ", Reflets : revue d'intervention sociale et communautaire, Vol. 6, № 1, p. 36-63.

TROVEPO (2014). La Petite histoire de la Table ronde des OVEP de l'Outaouais (TROVEPO) - 19732013 - 40 de luttes, réf. du 22 septembre 2015, http://trovepo.org/documents/ LaPetitehistoiredelaTROVEPO-versionimprim\%C3\%A9e.pdf 